\title{
Verifying Information Flow Goals in Security-Enhanced Linux*
}

\author{
Joshua D. Guttman Amy L. Herzog John D. Ramsdell \\ Clement W. Skorupka \\ The MITRE Corporation \\ guttman, aherzog, ramsdell, ragnor@mitre.org
}

July 28, 2004

\begin{abstract}
In this paper, we present a systematic way to determine the information flow security goals achieved by systems running a secure O/S, specifically systems running Security-Enhanced Linux. A formalization of the access control mechanism of the SELinux security server, together with a labeled transition system representing an SELinux configuration, provides our framework. Information flow security goal statements expressed in linear temporal logic provide a clear description of the objectives that SELinux is intended to achieve. We use model checking to determine whether security goals hold in a given system. These formal models combined with appropriate algorithms have led to automated tools for the verification of security properties in an SELinux system. Our approach has been used in other security management contexts over the past decade, under the name rigorous automated security management.
\end{abstract}

\section{Introduction}

In the 1980s, most of the rigorous work in information security was focused on operating systems, but the 1990s saw a strong trend toward network and distributed system security. The difficulty of having an impact in securing operating systems was part of the motivation for this trend.

There were two major obstacles. First, the only operating systems with significant deployment were large proprietary systems. Superimposing a security model and gaining assurance that the implementation enforced the model seemed intractable [10]. Second, the prime security model [1] was oriented toward preventing disclosure in multi-level secure systems [2], and this required

${ }^{*}$ This work was funded by the United States National Security Agency. Forthcoming in Journal of Computer Security. 
ensuring that even Trojan horse software exploiting covert channels in the system's implementation could compromise information only at a negligible rate. This was ultimately found to be unachievable [15].

These obstacles seem more tractable now. Open-source secure operating systems are now available, which are compatible with existing applications software, and hence attractive for organizations wanting more secure platforms for publicly accessible servers. Security-Enhanced Linux (SELinux) in particular offers well thought out security services $[8,9,11]$.

Moreover, a less stringent model of security, not focused on covert channels, is now relevant. The Orange Book's [2] focus on covert channels followed from a particular choice of threat model. It assumed that evaluated products would be used as general purpose computing systems, in which new software would be compiled and installed, so that the trusted computing base would include the operating system but no application software. In the long run, application software would belong to the adversary. Therefore, security goals such as confidentiality had to be achieved even if application software contained Trojan horses and attempted to signal secrets through covert channels.

In the bulk of cases today, by contrast, this is an inappropriate threat model. Instead, the system's security goals will be achieved by a combination of the operating system and its configuration data, together with specific pieces of application software interacting with critical system resources. Commonly, a network server for instance must service unauthenticated clients (as in retail electronic commerce), or must provide its own authentication and access control for its clients (as in a database server). Sensitive resources must reside on the same server so that transactions can complete. In this situation, only a small number of programs should be allowed to manipulate the sensitive resources. These special programs must be trustworthy, since there is no hope of acceptable system behavior otherwise. Access control can prevent these executables from being replaced or altered.

A security analysis in this context involves two different ingredients. One is understanding the operating system and how its policy is configured. The other is understanding the security critical aspects of the application software. While substantial work has been accomplished in developing techniques for determining the security relevant behavior of code (see [14] for a survey), many aspects of the problem remain to be studied. In this paper, however, we focus exclusively on the former ingredient. In particular, to make progress we must remove the issue of covert channels, which were unpreventable at the operating system level. Instead, we focus our attention on a context where programs interacting directly with the resources have a degree of trust, and the system owner aims to protect the confidentiality and integrity of sensitive resources manipulated by the programs.

Protection of sensitive resources may involve both integrity and confidentiality, which we represent as information flow security goals. These multi-step security goals say that information flowing between particular endpoints must traverse specific trustworthy programs along its path. To preserve integrity, each causal chain of interactions leading from untrusted sources to sensitive destina- 


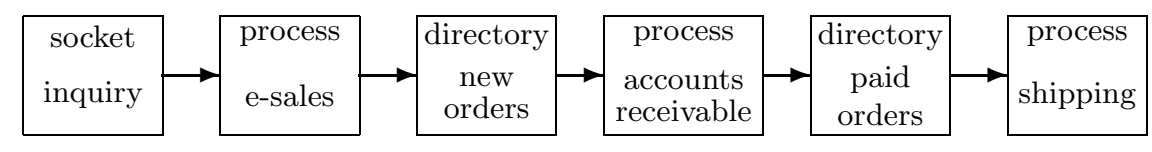

Figure 1: An E-Commerce Processing System

tions must traverse a program considered trusted to engage only in legitimate transactions with that destination. Dually, to preserve confidentiality, causal chains leading from sensitive sources to untrusted destinations must traverse a program trusted to filter outbound data. The trustworthy program determines what data can be released to the untrusted destination.

As an example focused on integrity, consider the e-commerce processing system described in Figure 1. In this scenario, orders are submitted by customers through an SSL-protected network socket at the left. An e-sales program ensures that a customer order is properly formatted, and if so, that the purchase prices for the different items are correct. For simplicity, we let the program write accepted orders to a file in a directory meant for new orders. Files in this directory will be read by an accounts receivable program, which after some online interaction with a credit card clearing house, causes the company's account to be credited. The order may now be written to the directory for paid orders. The shipping department program then checks inventory and causes the order to be shipped as soon as the goods are available.

The company wants to ensure that orders with erroneously low prices cannot arrive at accounts receivable, and that unpaid orders cannot arrive at the shipping department. The list of new orders (with prices) and the list of paid orders (used by the shipping process) are the sensitive resources in this example; the e-sales and accounts receivable processes must carefully control access to them.

In this paper, we aim at three goals. First, we develop a highly abstract model of the SELinux operating system access control mechanism. In this model, the system configuration determines a labeled transition system representing possible information flows (Section 2). Second, we propose a diagram-like way to state security goals, and give meaning to diagrams like Figure 1 using temporal logic (Section 3). Third, we describe briefly how to determine, using model checking, whether a goal is enforced by a particular configuration (Section 4). We regard this as a kind of rigorous, automated security management (Section 5, cf. [5]).

\section{An SELinux Model}

In this section, we will introduce the core ideas SELinux uses for access control (Section 2.1), after which we will introduce five relations that will summarize the contents of an SELinux system access control configuration file (Section 2.2). Section 2.2 describes how these relations are defined from the contents of a configuration file. The authorization relation (Section 2.3) synthesizes informa- 
tion from the five relations to determine which actions are allowed and which are prohibited. The flow relation then expresses what entities are affected by permissible actions (Section 2.4).

By a resource, we mean an entity such as a process, a file, a file descriptor, a socket, and so on, which is one of the entities considered when an action is adjudicated. We call the process making the system call the source resource, and the other resource at issue is the target resource. The target resource could also be a process, for instance in the case of an inter-process communication.

If an action is permitted, does the state of the process requesting the action change to reflect the state of a resource accessed, as is the case with a file read; or does the state of the resource change to reflect that state of the process, as is the case with a file write? In the former case, information flows from target resource to source resource. In the latter case, it flows the opposite direction, from source to target.

\subsection{Underlying Ideas of SELinux Access Control}

The SELinux security server makes decisions about system calls. For instance, when a process makes a write system call to write data to a particular file, the security server adjudicates this to see whether it should be permitted. Depending on the system call, it may need to check that several separate requirements are satisfied. In this case of writing to a file, the process must be permitted to modify that file. It must also be permitted to modify the file attributes of the file descriptor, which is a data structure containing a modification time that must be updated. If either of these checks fails, then the system call will not complete normally. We will call each of these checks a control requirement. In determining whether a control requirement is satisfied, the security server always considers the process requesting the system call and a resource on which the process is operating. In the case of a write, the relevant target resource for one control requirement is the file itself, while the other is the file descriptor.

In making its decision, the security server considers only a summary of the security relevant status of these resources; this summary is a data structure called a security context. Thus, for each system call, the security server evaluates one or more control requirements on the security context of a source and a target, and different targets may be relevant for different control requirements of the same system call.

Each control requirement is labeled by a pair. The first component is called the class of the control requirement. This is a value such as file for the file write control requirement, or $f d$ for the file descriptor modification control requirement. It indicates what kind of an entity the target resource is. Other possibilities include process, filesystem, and socket. The second component of a control requirement is called a permission, and it indicates what sort of an action is being adjudicated. In the case of writing data to a file, the two control requirements under consideration are

$$
\text { file, write and fd, setattr }
$$


checking whether the calling process is permitted to write the data to the file and whether it is permitted to set an attribute in the file descriptor (namely the modification time).

Each resource has its security context, which is the summary of its security relevant status delivered to the security server to use in adjudicating control requirements. A security context is a tuple consisting of three components, ${ }^{1}$ called a type, a role, and a user. The user is similar in intent to the normal Unix notion of user, and represents the person on behalf of whom the system is executing a process or maintaining a resource. The role, derived from the literature on role-based access control, is an intermediate notion intended to connect a collection of users with a corresponding collection of programs that they should be permitted to execute. Associated with the user component is a specification of the roles that user is permitted; the role then in turn specifies what types of processes those users are permitted to execute.

The most important component is the type, accounting for at least 22,000 out of the 22,500 access control statements in the sample policy file contained in the distribution. The type is used to specify the detailed interactions permitted between processes and other resources. For each system call, zero or more control requirements must be authorized; for each of these control requirements, the SELinux system will check that the type of the process is allowed to engage in this action against the type of the target. If any of these checks fails, then the system call will terminate before the kernel causes the corresponding change in system state.

For instance, in order to read a file, a process must be permitted to engage in the file read action against it. However, the read system call also causes an update to the attributes associated with the file descriptor, indicating the current time as the last time of file access. Thus, it must also be permitted to engage in the fd setattr action.

The exec system call has the most complex control requirements; they are summarized in Table 1. In the first three lines, the source type is the type of the process when it initiates the exec system call. In the fourth and fifth line, the source type is the type the resulting process will have in case the exec call succeeds, which may be different since part of the functionality of an exec call may be a change in security context. In the last line, the type of the parent process is considered, because the type of the new process must be one the parent is permitted to trace, in case the parent has been tracing this process. The targets of the control requirements are other objects involved in the exec call: the directories that must be searched to follow the path to the file; the file containing the binary image with which to overwrite memory; and the new process itself, which the old process must be permitted to launch. SELinux thus allows very tight control over which processes can execute which files, with which types of process resulting.

\footnotetext{
${ }^{1}$ or four components, if the system is compiled with support for multi-level security as it can be, but normally is not. For definiteness, we will assume MLS support is not compiled into the kernel in the remainder of this paper, although the approach we describe is equally applicable if it is.
} 


\begin{tabular}{|l|l|l|l|l|}
\hline \multirow{2}{*}{ Call } & \multicolumn{2}{|c|}{ Action } & Source & Target \\
\cline { 2 - 5 } & Class & Permission & (Process) & (Resource) \\
\hline execve & dir & search & Current & Path \\
& file & execute & Current & File \\
& process & transition & Current & New \\
& process & entrypoint & New & File \\
& fd & inherit & New & FD \\
& process & ptrace & Parent & New \\
\hline
\end{tabular}

Table 1: Actions in an exec System Call

It will be convenient to refer to the set of all types as $T$, the set of all roles as $R$, the set of all users (i.e. user names) as $U$, the set of all classes as $C$, and the set of all permissions as $P$. Because not all permissions make sense with all classes, we write $\Gamma \subset C \times P$ for the set of class-permission pairs that are used in SELinux.

A type $t \in T$ is called a domain if $t$ is the type of any process; we write $D \subset T$ for the set of domains. All roles but one are used to constrain the association of users with the types of processes. The dummy role $r_{o}$ (object_r) is used in security contexts where the type is not a domain.

\subsection{Syntax and Semantics for the Configuration File}

The configuration file defines several relations, five of which are of interest to us. The others concern auditing and other issues that not related to informationflow security goals. Each relation is built up by statements contained in the same configuration file. We will describe the syntax of these statements, and the relations that they are determining. The relations are gathered in Table 2.

Types are declared with statements such as

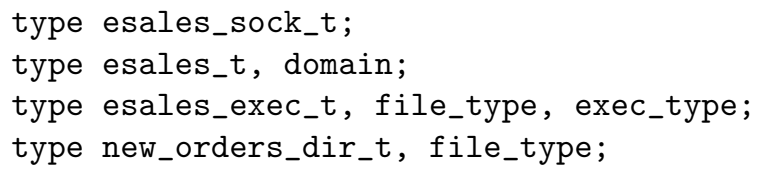

Any names after the first comma are attribute declarations, which declare that the new type belongs to a set named by the attributes. For instance, the domain attribute of esales_t stipulates that it belongs to the set of domains $D$. Attributes may be used in other configuration statements to refer to the set of all types having that attribute.

For each type, some actions are specified that processes executing with that type are allowed to perform. In the SELinux configuration file they are introduced by the keyword allow. For a request to succeed, some allow statement in the configuration file must authorize it. Each allow statement

allow $T_{s} T_{t}: C_{a} P_{a}$; 


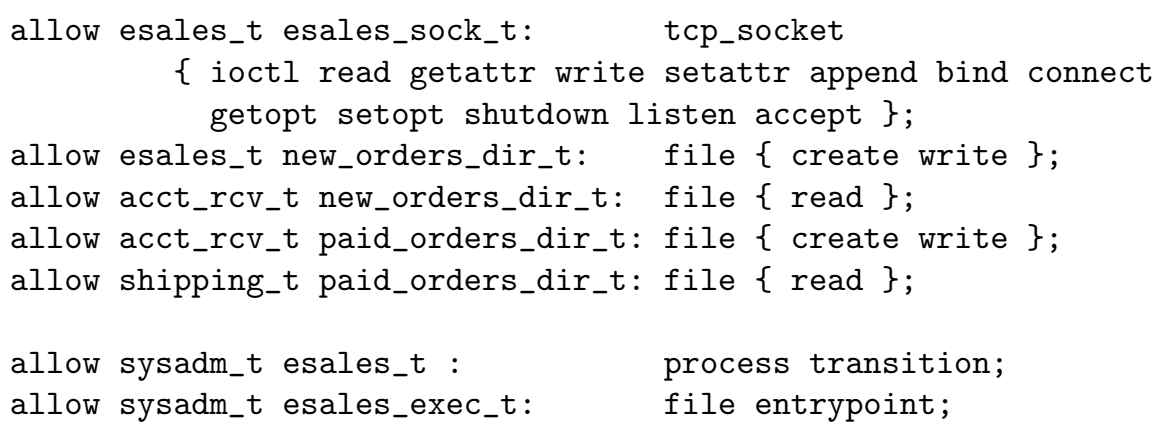

Figure 2: Electronic Commerce Example Allow Statements

specifies a set of process (source) types $T_{s}$, a set of resource (target) types $T_{t}$, a set of classes $C_{a}$, and a set of permissions $P_{a}$. We will not be specific about the syntax with which the sets of symbols such as $T_{s}, T_{t}$ etc. are presented. If a process whose type is in $T_{s}$ requests an action with a class-permission pair in $C_{a} \times P_{a}$ against a resource with type in $T_{t}$, then that request is authorized.

The allow statements for the electronic commerce example are gathered in Figure 2. The last two lines in the figure allow a process executing with type sysadm_t to initiate the esales program, transitioning to the new type esales_t, assuming that the esales executable is a binary image contained in some disk file of type esales_exec_t.

If the keyword self occurs as a target type, then it is treated specially. It indicates that each source type is allowed to engage in the specified actions with itself. For instance, a process of a particular type may be permitted to continue with the same type after executing a file of a some particular type.

When the keyword self is present, the statement implies that the tuple $(t, t, c, p) \in \alpha$ for each $t \in T_{s}, c \in C_{a}, p \in P_{a}$. Letting $T_{t}^{\prime}=T_{t} \backslash\{$ self $\}$, then the statement always implies $T_{s} \times T_{t}^{\prime} \times C_{a}, P_{a} \subset \alpha$. The relation $\alpha$ is the smallest relation compatible with the allow statements and these two rules.

The role allow statement takes the form

$$
\text { allow } R_{c} R_{n} \text {; }
$$

It controls change of role when a process has a transition from one security context to another; if the current role is in $R_{c}$ and the new role is in $R_{n}$, then this change of role will be permitted. More precisely, the statement specifies that $R_{c} \times R_{n} \subset \alpha_{\rho}$. The relation $\alpha_{\rho}$ is the smallest relation compatible with the role allow statements and this rule.

A role $r$ is declared with the following syntax.

$$
\text { role } r \text { types } T_{r} \text {; }
$$

In addition to declaring the role name $r$, the statement stipulates a set of types with which $r$ is permitted to be associated. The same role may be declared repeatedly to allow a large set of types to be declared conveniently. The statement 
$\alpha\left(t_{1}, t_{2}, c, p\right)$ is the type permission relation. It holds if $t_{1} \in D, t_{2} \in T$, and $(c, p) \in \Gamma$ for some allow statement in the configuration.

$\alpha_{\rho}\left(r_{1}, r_{2}\right)$ is the role transition relation. When a process changes security context, the role may change, but the old and new roles must satisfy $\alpha_{\rho}$.

$\rho(r, t)$ is the role-type relation. Each process in the system must have a security context such that $\rho(r, t)$ holds.

$\mu(u, r)$ is the user-role relation. Each process in the system must have a security context such that $\mu(u, r)$ holds.

$\chi_{c, p}\left(t_{1}, r_{1}, u_{1} ; t_{2}, r_{2}, u_{2}\right)$ is the constraint relation. Whenever $c, p$ is requested, the system checks that the constraint $\chi_{c, p}\left(t_{1}, r_{1}, u_{1} ; t_{2}, r_{2}, u_{2}\right)$ holds between the process security context and the resource security context. Constraints may be used to ensure that only privileged types of process change the user or role of existing resources, for instance.

Table 2: Access Control Relations

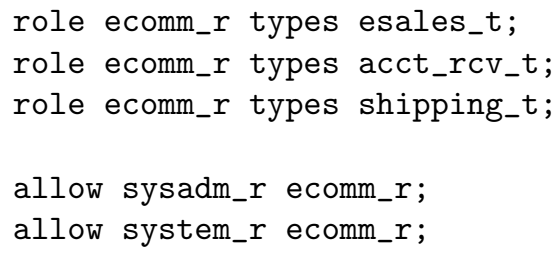

Figure 3: Electronic Commerce Example Role and Role Allow Statements

specifies that $\{r\} \times T_{r} \subset \rho$. The relation $\rho$ is the smallest relation compatible with the role declarations, the convention about $r_{o}$ mentioned at the end of Section 2.2, and this rule. The role declarations and role allow statements for the electronic commerce example are contained in Figure 3.

A user $u$ is declared with the following syntax.

$$
\text { user } u \text { types } R_{u} \text {; }
$$

In addition to declaring the user name $u$, the statement stipulates a set of roles with which $u$ is permitted to be associated. The same user may be declared repeatedly to allow a large set of roles to be declared conveniently. The statement specifies that $\{u\} \times R_{u} \subset \mu$. The relation $\mu$ is the smallest relation compatible with the role declarations and this rule. No users need be added in the electronic commerce example.

We express the special status of the dummy object role by stipulating that $\rho\left(r_{o}, t\right)$ holds whenever $t$ is not a domain, and $\mu\left(u, r_{o}\right)$ holds for all $u$.

Constraint definitions specify additional limits on transitions with the fol- 
lowing syntax.

$$
\text { constraint } C_{c} P_{c} \delta
$$

The constraint expression $\delta$ has a natural translation as a set of pairs of security contexts $\bar{\delta}$. Since a security context is a triple consisting of a type, a role, and a user, $\bar{\delta} \subset T \times R \times U \times T \times R \times U$, where $T, R, U$ are the sets of all types, roles, and users respectively. The value $\chi_{c, p}$ is the intersection of all $\bar{\delta}$ where $\delta$ is declared as a constraint on $C_{c}, P_{c}$ where $c \in C_{c}$ and $p \in P_{c}$. In effect, this means that all relevant constraints must hold true for an action to occur. No constraints need be added in the electronic commerce example.

We assume $\chi_{c, p}$ is empty when $(c, p) \notin \Gamma$.

\subsection{The Authorization Relation}

Our formal model of the SELinux authorization mechanism puts these five relations together in a specific way. The class-permission pair $c, p$ is authorized for a process with security context $t_{1}, r_{1}, u_{1}$ against a resource $t_{2}, r_{2}, u_{2}$ if:

$$
\begin{aligned}
& \alpha\left(t_{1}, t_{2}, c, p\right) \\
& \wedge \quad \rho\left(r_{1}, t_{1}\right) \quad \wedge \rho\left(r_{2}, t_{2}\right) \\
& \wedge \quad \mu\left(u_{1}, r_{1}\right) \quad \wedge \mu\left(u_{2}, r_{2}\right) \\
& \wedge \quad \chi_{c, p}\left(t_{1}, r_{1}, u_{1} ; t_{2}, r_{2}, u_{2}\right) \\
& \wedge \text { if } c=\text { process } \wedge p=\text { transition then } \alpha_{\rho}\left(r_{1}, r_{2}\right) \text {. }
\end{aligned}
$$

This relation $\Delta_{c, p}\left(t_{1}, r_{1}, u_{1} ; t_{2}, r_{2}, u_{2}\right)$ is the SELinux authorization predicate. The role allow relation $\alpha_{\rho}$ is relevant only in a single special case, namely when a process is making a transition to a new security context.

\subsection{The Information Flow Relation}

Some events (file write, for instance) transfer information from process to resource, while others (file read, for instance) transfer it from resource to process. SELinux has a file that describes how each $c, p$ transfers information, whether like a read, like a write, in both directions, or in neither. Information flows from an entity with security context $t, r, u$ to an entity with security context $t^{\prime}, r^{\prime}, u^{\prime}$ if for some event $c, p$ either

$$
c, p \text { has write-like flow and } \Delta_{c, p}\left(t, r, u ; t^{\prime}, r^{\prime}, u^{\prime}\right)
$$

or else

$$
c, p \text { has read-like flow and } \Delta_{c, p}\left(t^{\prime}, r^{\prime}, u^{\prime} ; t, r, u\right) \text {. }
$$

$\Phi_{c, p}\left(t, r, u ; t^{\prime}, r^{\prime}, u^{\prime}\right)$ means that at least one of these conditions holds, hence that there is flow from context $t, r, u$ to context $t^{\prime}, r^{\prime}, u^{\prime}$ through event $c, p$.

The file defining the direction of flow for each class-permission pair contains only a simple approximation. It does not take into account indirect flows caused by error conditions or variations in timing, and it does not consider flow 
into other system resources besides the process requesting the event and the resource against which the event is requested. This is why our analysis avoids the subtleties of covert channels.

Having defined the information flow relation $\Phi_{c, p}\left(t, r, u ; t^{\prime}, r^{\prime}, u^{\prime}\right)$, we regard it as a transition relation and consider what can be expressed in standard temporal logic in terms of this transition relation.

Because we want to work in linear temporal logic, where there are states and transitions but the transitions have no labels, we digest the label $c, p$ into the state before the transition. Thus, we regard a state as a sextuple $\langle t, r, u, c, p, k\rangle$ consisting of a type, user, and role, as well as a class and permission signifying the transition about to occur.

The last component $k$ is a Boolean flag used to make the transition relation total. In linear temporal logic, there is always a next state in the sense that e.g. $\square$ true is valid. Since there may not be any $t^{\prime}, r^{\prime}, u^{\prime}$ such that $\Phi_{c, p}\left(t, r, u ; t^{\prime}, r^{\prime}, u^{\prime}\right)$, we need the flag $k$ to indicate when the transition relation is diverging from $\Phi$.

When $k$ is true, it indicates that all transitions so far have been legitimate. When $k$ is false, some bogus transition has occurred, and the states no longer bear any meaningful relation to $\Phi$.

If $k$ is false, then in the next state $k$ must remain false, although the remaining components can take any value. If $k$ is true, then $k^{\prime}$ is true in the next state only when the type, user, and role are values $t^{\prime}, r^{\prime}, u^{\prime}$ such that $\Phi_{c, p}\left(t, r, u ; t^{\prime}, r^{\prime}, u^{\prime}\right)$; the next $c^{\prime}, p^{\prime}$ is unconstrained. Otherwise, $k^{\prime}$ is false. Thus, the transition relation is highly non-deterministic.

The initial states $\langle t, r, u, c, p, k\rangle$ for this model are the ones that are compatible with $\rho$ and $\mu$ in that

$$
\rho(r, t) \wedge \mu(u, r) .
$$

$\Phi$ says that there is a causal effect of one state on the next, and iterated applications of the relation say that there is some sequence of events (possibly involving many different processes and resources) creating a causal chain from the first state of the sequence to the last.

Suppose then that there is a sequence of events

$$
\left(t_{0}, r_{0}, u_{0}\right) \stackrel{c_{0}, p_{0}}{\longrightarrow}\left(t_{1}, r_{1}, u_{1}\right) \stackrel{c_{1}, p_{1}}{\longrightarrow} \ldots \stackrel{c_{n-1}, p_{n-1}}{\longrightarrow}\left(t_{n}, r_{n}, u_{n}\right)
$$

in which $\Phi_{c_{j}, p_{j}}\left(t_{j}, r_{j}, u_{j} ; t_{j+1}, r_{j+1}, u_{j+1}\right)$ for each $j$ from 0 to $n-1$. Then equivalently, we have an execution history in which the transition relation holds between $\left(t_{j}, r_{j}, u_{j}, c_{j}, p_{j}\right.$, true) and $\left(t_{j+1}, r_{j+1}, u_{j+1}, c_{j+1}, p_{j+1}\right.$, true $)$ for each $j$ from 0 to $n-1$. Here $c_{n}, p_{n}$ is unconstrained. In this case, we say that there is an information flow from $\left(t_{0}, r_{0}, u_{0}\right)$ to $\left(t_{n}, r_{n}, u_{n}\right)$ via the sequence of control requirements $\left\langle\left(c_{0}, p_{0}\right), \ldots,\left(c_{n-1}, p_{n-1}\right)\right\rangle$.

For instance, suppose $n=2$ and $\left\langle\left(c_{0}, p_{0}\right),\left(c_{1}, p_{1}\right)\right\rangle$ is $\langle($ file,write $),(f i l e$, read $)\rangle$. In this case, the flow consists of the fact that a process with type $t_{0}$ can write to files with type $t_{1}$, while processes with type $t_{2}$ can read from them. Thus, information flows from $t_{0}$ to $t_{2}$ via file write and read. Alternatively, suppose 
that $n=2$ and $\left\langle\left(c_{0}, p_{0}\right),\left(c_{1}, p_{1}\right)\right\rangle$ is $\langle(\mathrm{fd}$, setattr $)$, (fd, getattr $\left.)\right\rangle$. Then, information flows through manipulating the attributes of a file descriptor, which the first process can set while the second process gets them; modification time may be the attribute in question. Thus, a path through the relation $\Phi$ or through the transition relation defines a sequence of events through which some causal effects are transmitted from the first security context to the last.

The model we have just developed, and encoded in the information flow predicate $\Phi_{c, p}$, is an enormous simplification of the SELinux system. It concentrates on the information flow consequences of individual events, and abstracts from all aspects of system resources apart from their security contexts. The benefit of this approach is to provide a minimal representation still allowing us to analyze core security goals achieved by an SELinux configuration.

It is the purpose of a security policy to say which of these causal paths are permissible. All others are prohibited. The policy may classify flow according to the initial and final security contexts; according to the intermediate contexts, and according to the control requirements lying on the path.

\section{Security Goals}

The SELinux access control mechanism allows the policy writer to protect the confidentiality and integrity of sensitive resources. As described above, protecting these resources entails ensuring that information flowing from one place to another must traverse specific points along its path. Security goals of this sort are examples of intransitive noninterference: information flow from one security context to another is only acceptable if it happens through another, trustworthy, program [13]. These intransitive noninterference chains cover realistic security desires, ranging from the simple to the complex.

Consider the case of raw disk access. Since directly accessing the disk bypasses traditional access controls, it is likely that a system administrator would want only specific administrative programs to have the ability. In the sample SELinux policy, raw disk data has the type fixed_disk_device_t, and the type $\mathrm{fsadm}_{-} \mathrm{t}$ is used for administrative programs requiring direct disk access. The system administrator aims to ensure that all one-step paths ending in fixed_disk_device_t begin with fsadm_t. This is the simplest form of causal chain.

Web servers provide slightly more complex security needs. When running a web server, it is very important to keep user capabilities separate from server administrator capabilities. Since frequently the administrator is also a user on the system, the core desire is to prevent users from performing sensitive operations without first supplying (for example) the administrator password. A concrete example from the sample SELinux policy involves the modification and execution of web server system scripts. A special domain, httpd_admin_t, is defined for web server administration functions. Thus in this case, an administrator wishes to ensure that any path of information flow starting at user_t and ending in a write to scripts of the type httpd_sys_script_t passes through 
httpd_admin_t.

A still more complex security goal is pictured in the e-commerce example in Figure 1. This pictures an integrity goal: although untrusted users may connect to the server, only paid orders should be shipped. A process with SELinux type esales_t checks new orders read from a resource with type esales_sock_t; after being checked they are written to a file with type new_orders_dir_type. The accounts receivable program has type acct_rcv_t, and writes out paid orders to files with type paid_orders_dir_t, which are readable by a process having type shipping_t.

\subsection{Visualizing Causal Chains: Diagrams}

We will now formalize a particular way to express information flow goals. We wish to ensure that all paths through a system from a starting security context to a final security context go through a series of intermediate steps. These intermediate steps can be viewed, as in Figure 1, as security contexts or sets of them. We may also sometimes wish to specify the means by which one security context can affect another: in other words, we may wish to label the arrows in Figure 1 with class-permission pairs such as socket read, file create, file write, etc. Information flow security goals are expressed in this alternating chain of security contexts and actions.

When constructing a chain, one has four degrees of freedom. First, one can define what security contexts appear at a stage in the process; formulas defining these sets use symbols such as $\sigma_{i}$. We adopt the convention of writing " $\sigma_{i}$ is $\phi$ " where $\phi$ involves only the variables $t, r, u$ free, to mean that $\sigma_{i}$ is defined to be the set $\{(t, r, u) \mid \phi\}$. For instance, for the web server integrity goal described above,

$$
\begin{aligned}
& \sigma_{0} \text { is } t=\text { user_t } \\
& \sigma_{1} \text { is } t=\text { httpd_admin_t } \\
& \sigma_{2} \text { is } t=\text { httpd_sys_script_t. }
\end{aligned}
$$

Second, one may characterize what actions or events may transfer information from one context to the next; formulas defining these sets use symbols such as $\gamma_{i}$. Again, we adopt the convention of writing " $\gamma_{i}$ is $\phi$ " where $\phi$ involves the variables $c, p$, to mean that $\gamma_{i}$ is defined to be the set $\{(c, p) \mid \phi\}$. Members of $\gamma_{i}$ are SELinux class-permission pairs. Following our web server example goal,

$$
\begin{aligned}
& \gamma_{0} \text { is true } \\
& \gamma_{1} \text { is } c=\text { file } \wedge p \in\{\text { write, append }\}
\end{aligned}
$$

The third kind of freedom captures the intuitive notion of the length of the arrows. Between two security contexts in our causal path, we may be interested in constraining the paths to a single event. (Our raw disk access example concerns only direct disk accesses, rather than longer chains.) Longer paths between contexts may also be of interest, however. The e-commerce example could provide a concrete case: Perhaps some customers receive a special discount on their order, which may need to be checked between the e-sales program and the 
new orders file. Thus, flow from esales_t to new_order_type may go by way of a process with a different type that validates the discount, and possibly other intermediate types.

Our web server security goal is also concerned with longer paths, since it would be wise to consider all information flows from a user ending in the writing of HTTP system scripts.

We distinguish such iterated events by surrounding them with square brackets and a superscript + . Let $\lambda_{i}$ be a label of one of the forms $\gamma_{i}$ or $\left[\gamma_{i}\right]^{+}$. The final action formulas for our web server example are the following:

$$
\begin{aligned}
& \lambda_{0} \text { is }[\text { true }]^{+} \\
& \lambda_{1} \text { is }[c=\text { file } \wedge p \in\{\text { write, append }\}]^{+} .
\end{aligned}
$$

The fourth degree of freedom is that of exceptions, although we will return later to fill in this detail (see Section 3.2.3). Ignoring exceptions, we can write an information flow policy goal in the following visual form:

$$
\sigma_{0} \stackrel{\lambda_{0}}{\longrightarrow} \sigma_{1} \stackrel{\lambda_{1}}{\longrightarrow} \cdots \stackrel{\lambda_{n-2}}{\longrightarrow} \sigma_{n-1} \stackrel{\lambda_{n-1}}{\longrightarrow} \sigma_{n}
$$

Note the similarity between this form and our e-commerce example in Figure 1. Translating the picture in Figure 1 to our information flow formulas, we obtain the diagram shown in Figure 4. The $\sigma_{i}$ correspond directly to the boxes pictured in Figure 1. The labels $\lambda_{i}$ correspond to the arrows pictured there.

To see the relevance of the + here, consider that when a process reads from a socket, information flows forward from the socket to the process. When it sets socket options, information also flows backward to the socket. For this reason, there are also longer, cyclic paths between esales_sock_t and esales_t. The + sign permits longer paths in this case.

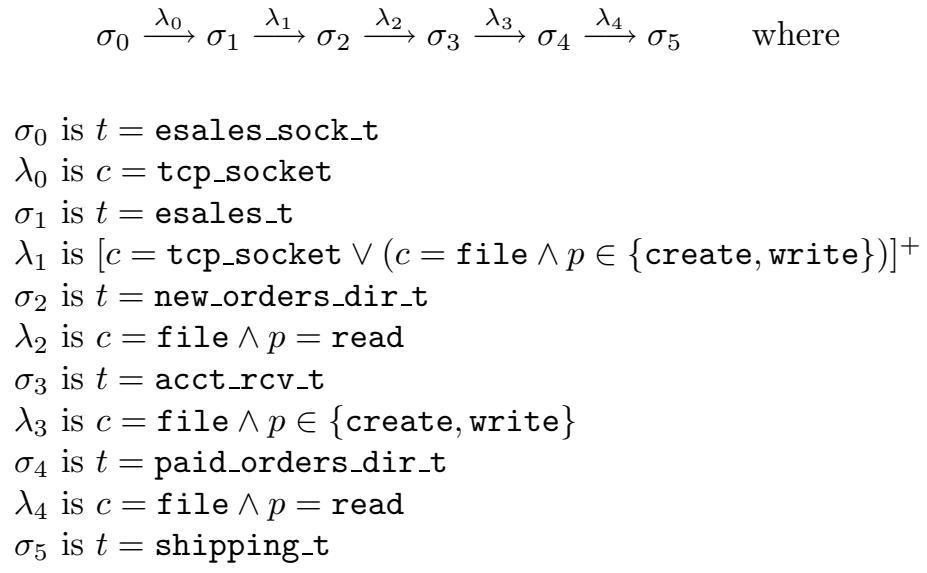

Figure 4: Security Diagram for the Electronic Commerce Example 


\subsection{Formalizing Diagrams in Linear Temporal Logic}

We interpret an information flow policy as an assertion about all sequences of state transitions leading from a state in $\sigma_{0}$ to a state in $\sigma_{n}$. It asserts that this path must encounter the $\sigma_{i}$ in the order given, executing events from $\lambda_{i}$ in each stage.

To formalize these assertions, we first represent the fact that they concern only state transitions leading from states in $\sigma_{0}$ to states in $\sigma_{n}$. We may express this as the hypothesis $H=\sigma_{0} \wedge \diamond\left(\sigma_{n} \wedge k\right)$, stating that $\sigma_{0}$ currently holds and $\sigma_{n}$ will eventually hold, and where $k$ is true because all transitions so far have satisfied $\Phi$. We interpret an information flow diagram (1) by two formulas of Linear Temporal Logic (LTL).

\subsubsection{Order Assertions}

The first formula asserts that states are encountered in the right order, subject to the hypothesis $H$ that we are passing from $\sigma_{0}$ to $\sigma_{n}$ :

$$
H \Rightarrow \bigwedge_{0<i<n} \sigma_{i} \mathcal{R} \neg \sigma_{i+1} .
$$

The operator $\mathcal{R}$ ("releases") asserts that its right hand operand is true and remains true until its left hand operator has been true at least once. Thus, this formula asserts that each set $\sigma_{i+1}$ is not encountered until after $\sigma_{i}$ has been encountered, along paths from $\sigma_{0}$ to $\sigma_{n}$.

\subsubsection{Event Assertions}

The other formula asserts that the transitions along a path from $\sigma_{0}$ to $\sigma_{n}$ proceed using the right class-permission pairs. From the time that $\sigma_{i}$ has been encountered but $\sigma_{i+1}$ has not yet been reached, all of the transitions should be in the set $\gamma_{i}$. In the case where none of the $\gamma_{i}$ are decorated with a + , this leads to the formula

$$
H \Rightarrow \gamma_{0} \wedge \mathcal{X}\left(\sigma_{1} \wedge \gamma_{1} \wedge \mathcal{X}(\cdots)\right) .
$$

$\mathcal{X} \phi$ asserts of a state that $\phi$ is true in the next state immediately after it. Thus, we start with a $\gamma_{0}$ which brings us to $\sigma_{1}$ and then continue with a $\gamma_{1}$ which brings us to $\sigma_{2}$ and so on. If all the $\gamma_{i}$ are surrounded by square brackets with $\mathrm{a}+$, then we want to say that a $\gamma_{i}$ occurs, and then $\gamma_{i}$ s continue until a $\sigma_{i+1}$ is reached, and so on:

$$
H \Rightarrow \gamma_{0} \wedge \mathcal{X}\left(\gamma_{0} \mathcal{U}\left(\sigma_{1} \wedge \gamma_{1} \wedge \mathcal{X}\left(\gamma_{1} \mathcal{U} \cdots\right)\right)\right)
$$

$\phi \mathcal{U} \psi$ is true in a state if $\psi$ eventually becomes true, and $\phi$ remains true until the first such occasion. We combine the two forms into a formula

$$
H \Rightarrow \gamma_{0} \mathcal{O}_{0}\left(\sigma_{1} \wedge\left(\gamma_{1} \mathcal{O}_{1}\left(\sigma_{2} \ldots\right)\right)\right) .
$$

When the label $\lambda_{i}$ is of the form $\gamma_{i}$, then $\phi \mathcal{O}_{i} \psi$ is defined to be $\phi \wedge \mathcal{X} \psi$. When the label $\lambda_{i}$ is of the form $\left[\gamma_{i}\right]^{+}$, then $\phi \mathcal{O}_{i} \psi$ is defined to be $\phi \wedge \mathcal{X}(\phi \mathcal{U} \psi)$. 
Formulas 2 and 3 do not need a leading "always" $\square$, because, for states such that $k$ is true, all accessible states are also initial states.

\subsubsection{Exceptions}

We now return to the fourth degree of freedom when constructing causal chains, that of exceptions. An example from our e-commerce case can provide motivation. Suppose there is a directory for status queries, with type query_t, such that flow from a network socket to the shipping department program is permitted if it comes by way of query_t. The e-commerce system designers intended for this to be acceptable, yet it provides a counterexample to the goals mentioned in Section 1. (There is then a corresponding security requirement on the shipping department program, stating that input from the status query files never cause products to be shipped, but verifying that requirement is a matter for programming language security analysis (see e.g. [14]) rather than operating system security analysis.)

The type query_t and class network socket are exceptions to the more general rule. In cases such as this, we want to make our assertions subject to these exceptions. If the exception occurs, we do not care what the information flow is; if the exception does not occur, then we want the information flow diagram to hold true as before. The exceptional condition may be either a state or a transition, that is, a class-permission pair. For instance, perhaps the accounts receivable program can send a signal to the shipping program to tell it when to stat the shared directory. This signal is a flow of information to shipping that does not traverse the type paid_orders_t. However, it is merely advisory, and we know it causes nothing to be shipped unless the program succeeds in reading a new paid order. Thus, there is no need to prohibit this flow.

We incorporate exceptions without changing the form of Equations 2 and 3. Instead, let $\sigma_{e}$ be the set of exceptional states, and let $\gamma_{e}$ be the set of exceptional transitions; we redefine $H$ to take the form:

$$
\sigma_{0} \wedge\left(\left(\neg \sigma_{e} \wedge \neg \gamma_{e}\right) \mathcal{U}\left(\sigma_{n} \wedge k\right)\right)
$$

Thus, we concern ourselves with a path only if it started at $\sigma_{0}$ and avoided $\sigma_{e}$ and $\gamma_{e}$ until reaching a state in which $\sigma_{n} \wedge k$. If a path is of this form, then we require that the bodies of Equations 2 and 3 hold. Taking into account exceptions, we can now write information flow policy goals in the following visible form:

$$
\sigma_{0} \stackrel{\lambda_{0}}{\longrightarrow} \sigma_{1} \stackrel{\lambda_{1}}{\longrightarrow} \cdots \stackrel{\lambda_{n-2}}{\longrightarrow} \sigma_{n-1} \stackrel{\lambda_{n-1}}{\longrightarrow} \sigma_{n} \quad\left[\sigma_{e} ; \gamma_{e}\right]
$$

As we will see in Section 4, exceptions become important very quickly when analyzing complex policies such as the policy included in the sample SELinux distribution. 


\section{Goal Enforcement and Implementation}

We have written software that reads and analyzes SELinux configuration files. It constructs a labeled transition system with security contexts as states, and actions as transition labels. The transition relation represents the information flow relation $\Phi_{c, p}$, and the initial states are the security contexts that satisfy $\rho(r, t) \wedge \mu(u, r)$. The labeled transition system is written to disk in an easily read format.

We have tools that use the labeled transition system to construct a Binary Decision Diagram (BDD) for specialized processing, but the tool most often used takes the labeled transition system along with a set of diagrams, and produces input for the model checker NuSMV [12].

Consider the web server diagram introduced in Section 3.1 and the policy configuration files in the NSA SELinux release. ${ }^{2}$ In this case, we use the diagram

$$
\begin{aligned}
& \sigma_{0} \stackrel{\lambda_{0}}{\longrightarrow} \sigma_{1} \stackrel{\lambda_{1}}{\longrightarrow} \sigma_{2} \quad\left[\sigma_{e} ; \lambda_{e}\right] \quad \text { where } \\
& \sigma_{0} \text { is } t=\text { user_t } \\
& \lambda_{0} \text { is }[\text { true }]^{+} \\
& \sigma_{1} \text { is } t=\text { httpd_admin_t } \\
& \lambda_{1} \text { is }[c=\text { file } \wedge p \in\{\text { write, append }\}]^{+} \\
& \sigma_{2} \text { is } t=\text { httpd_sys_script_t } \\
& \sigma_{e} \text { is false } \\
& \lambda_{e} \text { is false }
\end{aligned}
$$

To see if the default policy meets the security goal expressed by the diagram, we created the labeled transition system from the policy configuration files. We then encoded the diagram in a textual syntax, and used it and the labeled transition system to create input for NuSMV.

Figure 5 contains an excerpt of the output from NuSMV. It shows that the security goal is not met by the policy files. The model checker found an illegal path of information flow between four security contexts. Recall that a state used by the model checker includes the class and permission labeling the transition about to occur. Thus the first step in the path makes use of an allowed information flow that starts with security context (user_t,system_r,system_u), and goes to security context (netif_ipsec2_t,object_r),jdoe_u) via the netif_c rawip_send_p action.

The security goal is met by the sample policy files in the SELinux release if the diagram in Equation 5 above has the following exception for security contexts $\sigma_{e}$ :

$$
r=\operatorname{sysadm} \_r \vee r=\text { system } \_r
$$

The exception $\sigma_{e}$ means that any path that contains a type associated with the roles sysadm $\_r$ or system $\_r$ is ignored, and not considered a violation of the security goal. These roles are associated with system processes manipulating

\footnotetext{
${ }^{2}$ We have used release 2003040709 of April 2003 throughout this section.
} 


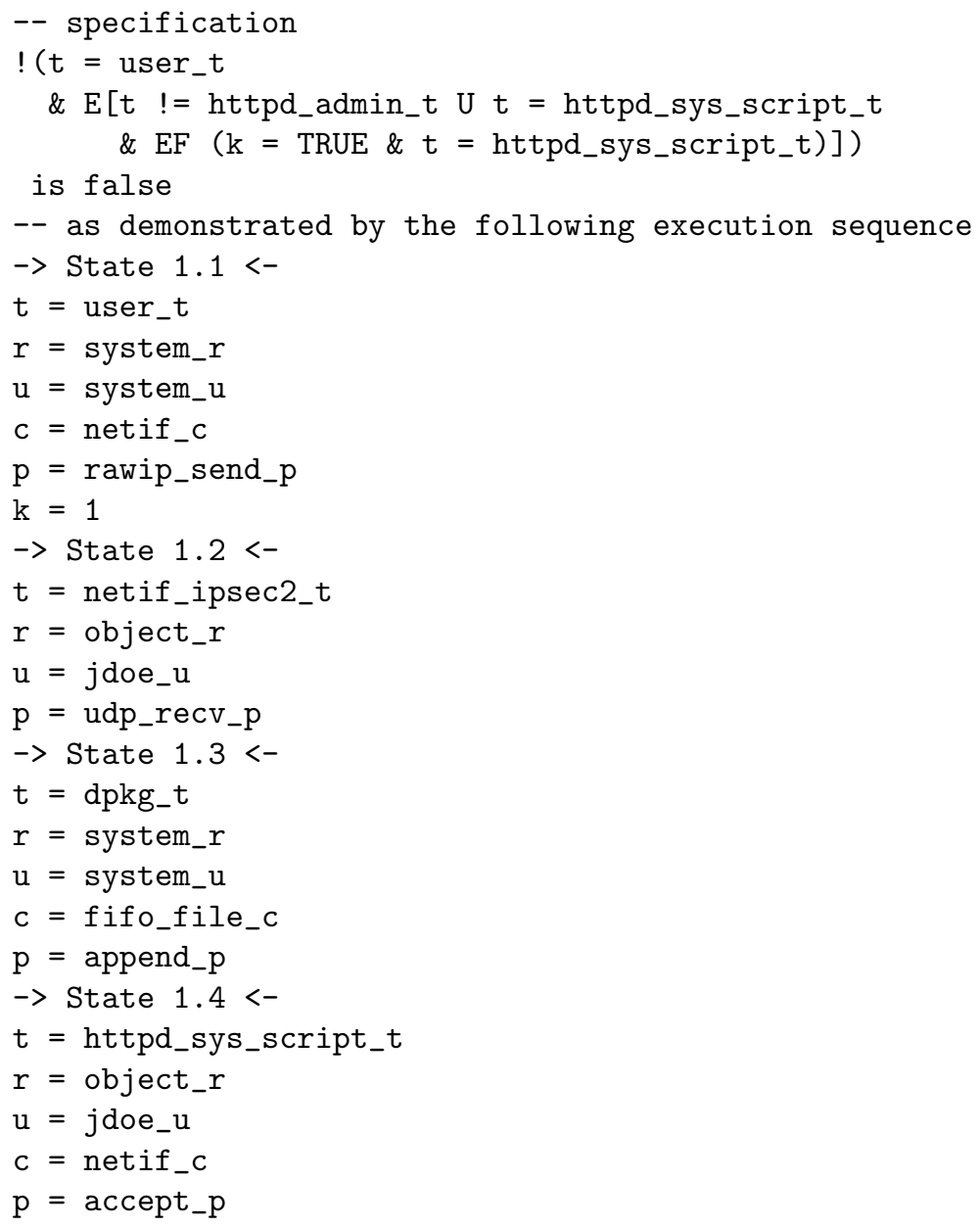

Figure 5: HTTPD Security Goal Failure 
low-level resources. Such processes can alter many aspects of the system, but are run only by purportedly trustworthy system administrators.

The diagram in Figure 4 can be used to specify a security goal for the e-commerce processing system in Figure 1, however, once again, the security context exception above must be added. The modified diagram expresses a security goal that is satisfied by policy files modified to include the additional types and permissions described in Section 2.2.

For policy files based on the ones in the release, NuSMV execution typically requires about $150 \mathrm{MB}$ of store, and about 10 minutes of $\mathrm{CPU}$ time on a $1 \mathrm{GHz}$ Intel GNU/Linux laptop. The resources required to construct the input for NuSMV are negligible.

The programs that transform SELinux configuration files and diagrams into NuSMV input are written in the OCaml dialect of ML [7]. The use of a functional language allowed an implementation that closely matches its mathematical specification.

\section{Rigorous Automated Security Management}

In this paper, we have presented a systematic way to analyze the information flow goals achieved by an SELinux system. A formalization of the access control mechanism of the SELinux security server together with a labeled transition system representing an SELinux configuration provides our framework. Security goal statements in linear temporal logic provide a clear description of the objectives that SELinux is intended to achieve. We use model checking to determine whether security goals hold in a given system.

The approach used in developing these formalizations and analysis methods has been used in other security management contexts over the past decade, most recently under the name rigorous automated security management $[3,4,5,6]$. This method front-loads the contribution of formal methods to problem-solving. The focus is on modeling devices, their behavior as a function of configurations, and the consequences of their interactions. A class of practically important security goals must also be expressible in terms of these models.

These models suggest algorithms taking as input information about system configuration, and returning the security goals satisfied in that system. In some cases, although not as yet in the case of SELinux, we can also derive algorithms to generate configurations to satisfy given security goals. The formal models provide a rigorous justification of soundness. By contrast, algorithms are implemented as ordinary computer programs requiring no logical expertise to use. Resolving practical problems then requires little time, and no formal methods specialists. Rigorous automated security management consists of four steps.

Modeling Construct a simple formal model of the problem domain. In this paper, we have seen the formalization of the access control mechanism of the SELinux security server, and the transition relation of an SELinux security policy. 
Security Goals SELinux is intended to achieve information flow security goals. These take the forms given in Equations 2 and 3.

Goal Enforcement The security goals and underlying model must be chosen so that there is an algorithm that, given a system as represented in the model, and a particular goal statement of one of the selected logical forms, determines whether the system satisfies that goal. In the SELinux system, model checking provides our assurance.

Implementation Having defined and verified one or several goal enforcement algorithms, one writes a program to check goal enforcement. The inputs to this program consist of goal statements that should be enforced, and system configuration information. In this paper, we have discussed an implementation based on NuSMV and mentioned our own specially adapted BDD software.

For systems such as SELinux, formal models of access control configuration and checking reasonable security goals are tractable. A combination of this formal model and an appropriate algorithm has led to automatic tools for the verification of security properties in an SELinux system. While much future work remains, we believe this approach to be an important step toward increasing the usefulness of secure operating systems.

\section{References}

[1] David E. Bell and Leonard J. LaPadula. Computer security model: Unified exposition and Multics interpretation. Technical Report 75-306, ESD, June 1975.

[2] Department of Defense trusted computer system evaluation criteria. DOD 5200.28-STD, December 1985.

[3] Joshua D. Guttman. Filtering postures: Local enforcement for global policies. In Proceedings, 1997 IEEE Symposium on Security and Privacy, pages 120-29. IEEE Computer Society Press, May 1997.

[4] Joshua D. Guttman. Security goals: Packet trajectories and strand spaces. In Roberto Gorrieri and Riccardo Focardi, editors, Foundations of Security Analysis and Design, volume 2171 of LNCS, pages 197-261. Springer Verlag, 2001.

[5] Joshua D. Guttman and Amy L. Herzog. Rigorous automated network security management. International Journal for Information Security, 2004. Forthcoming.

[6] Joshua D. Guttman, Amy L. Herzog, and F. Javier Thayer. Authentication and confidentiality via IPsec. In D. Gollman, editor, ESORICS 2000: European Symposium on Research in Computer Security, number 1895 in LNCS. Springer Verlag, 2000. 
[7] Xavier Leroy, Damien Doligez, Jacques Garrigue, Didier Rémy, and Jérôme Vouillon. The Objective Caml System. INRIA, http://caml.inria.fr/, 2000. Version 3.00.

[8] P. Loscocco and S. Smalley. Integrating flexible support for security policies into the Linux operating system. In Proceedings of the FREENIX Track of the 2001 USENIX Annual Technical Conference, 2001.

[9] P. Loscocco and S. Smalley. Meeting critical security objectives with security-enhanced Linux. In Proceedings of the 2001 Ottawa Linux Symposium, 2001.

[10] P.A. Loscocco, S.D. Smalley, P.A. Muckelbauer, R.C. Taylor, S.J. Turner, and J.F. Farrell. The inevitability of failure: The flawed assumption of security in modern computing environments. In Proceedings of the 21st National Information Systems Security Conference, pages 303-314, October 1998.

[11] National Security Agency. Security-enhanced Linux. At URL http://www . nsa.gov/selinux/index.html, April 2003.

[12] NuSMV: a new symbolic model checker. URL http://sra.itc.it/tools/ nusmv, 2001.

[13] A. W. Roscoe and M. H. Goldsmith. What is intransitive noninterference? In 12th IEEE Computer Security Foundations Workshop, pages 228-238. IEEE CS Press, June 1999.

[14] Andrei Sabelfeld and Andrew C. Myers. Language-based information-flow security. IEEE Journal on Selected Areas in Communication, 21(1):5-19, January 2003.

[15] John C. Wray. An analysis of covert timing channels. In Proceedings, 1991 IEEE Symposium on Research in Security and Privacy, pages 2-7. IEEE Computer Society, May 1991. 


\section{Contents}

1 Introduction 1

2 An SELinux Model 3

2.1 Underlying Ideas of SELinux Access Control . . . . . . . . . . . . 4

2.2 Syntax and Semantics for the Configuration File . . . . . . . . 6

2.3 The Authorization Relation . . . . . . . . . . . . . 9

2.4 The Information Flow Relation . . . . . . . . . . . . . . . 9

3 Security Goals $\quad 11$

3.1 Visualizing Causal Chains: Diagrams . . . . . . . . . . . . . . . 12

3.2 Formalizing Diagrams in Linear Temporal Logic . . . . . . . . . 14

3.2.1 Order Assertions ................. . . 14

3.2.2 Event Assertions . . . . . . . . . . . . . . . . . 14

3.2.3 Exceptions .................. 15

4 Goal Enforcement and Implementation 16

5 Rigorous Automated Security Management 18 